\title{
A Search For Dark Matter Signals in the Galactic Halo with HAWC
}

\author{
Corresponding authors: Joseph Lundeen ${ }^{* 1}$, J Patrick Harding ${ }^{2}$ \\ for the HAWC Collaboration ${ }^{\dagger}$ \\ ${ }^{1}$ Michigan Sate University \\ ${ }^{2}$ Los Alamos National Laboratory \\ $\dagger$ https://www.hawc-observatory.org/collaboration/icrc2019.php \\ E-mail: lundeenj@msu.edu, jpharding@lanl.gov
}

The High Altitude Water Cherenkov Observatory (HAWC) is a high energy (500 GeV to 100 $\mathrm{TeV})$ gamma ray detector located in central Mexico. HAWC has both a wide $(\approx 2 \mathrm{sr})$ field of view and near continuous duty cycle, making it ideal for unbiased sky surveys and analysis of highly extended sources. We make use of HAWC's survey abilities to search for dark matter signals originating from the Milky Way Galaxy. By taking an unbiased sample of the sky, we set characteristic upper limits on dark matter annihilation flux for various dark matter masses and annihilation channels as a function of declination. These characteristic limits are then combined with models of the dark matter spatial distribution in order to calculate the HAWC sensitivity to gamma-ray emission from both dark matter substructure and the main Galactic halo. We use this sensitivity to optimize a search for dark matter emission from the main Galactic halo.

36th International Cosmic Ray Conference -ICRC2019-

July 24th - August 1st, 2019

Madison, WI, U.S.A.

${ }^{*}$ Speaker. 


\section{Introduction}

\section{Dark Matter and Gamma Rays}

The expected differential photon flux (per unit energy) from a dark matter halo is described by the following equation:

$$
\frac{d \Phi}{d E}=\frac{J\langle\sigma v\rangle}{8 \pi M^{2}} \frac{d N(M, \text { channel })}{d E}
$$

where $\langle\sigma v\rangle$ is the velocity-weighted annihilation cross section and $M$ is the dark matter mass. The $J$-factor is defined as:

$$
J=\iint \rho_{d m}^{2}(l, \Omega) d l d \Omega
$$

an integral of the squared dark matter mass density profile over the line of sight and solid angle of the observation.

The quantity $\frac{d N}{d E}$ is the gamma-ray spectrum from a single dark matter annihilation [1]. We use PYTHIA 8.2 to calculate this function by simulating dark matter annihilation and recording the number of gamma rays produced; our models assume $100 \%$ branching ratios of dark matter into individual standard model particle channels [2]. We include the $b \bar{b}$ channel since this has been extensively studied in other experiments as well the $\tau^{+} \tau^{-}$channel because this is the heaviest solely leptonic channel available. An example of dark matter spectral shapes, showing the characteristic hard energy cut-off at the dark matter mass, is shown in Fig. 1.

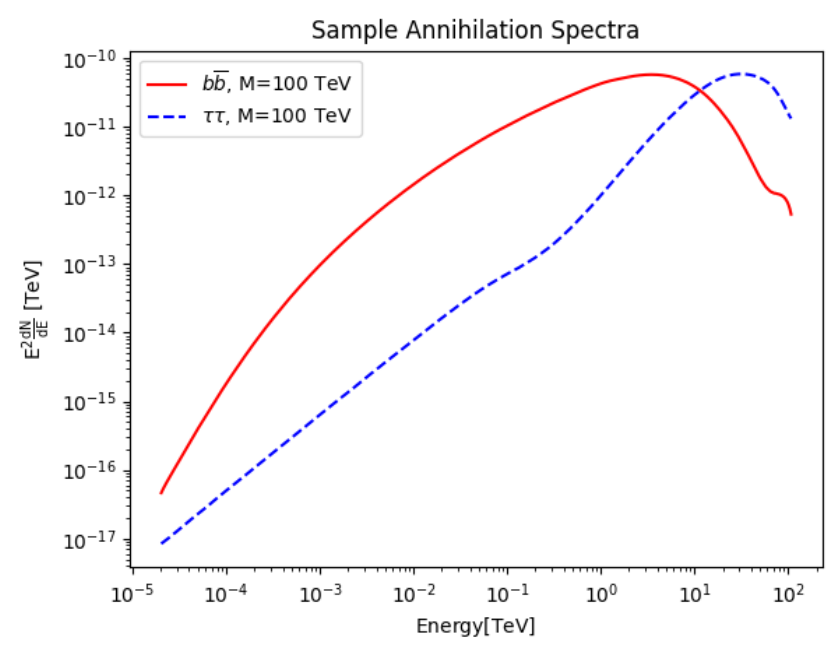

Figure 1: Example dark matter energy spectra for two annihilation channels $\left(b \bar{b}\right.$ and $\left.\tau^{+} \tau^{-}\right)$. The spectra assume a mass of $100 \mathrm{TeV}$ and cut off sharply at this energy. These are used in Eq. 2.1 and they show the same characteristic shape.

\section{Spatial Profiles}

The behavior of the Galactic Halo density profile is poorly constrained close $t \mathrm{o}$ the center. Evidence from numberical simulations supports Einasto density profile shown Eq. 3.1, which is 


\begin{tabular}{c|c|c|c}
$R_{\odot}(\mathrm{kpc})$ & $\rho_{\odot}\left(\mathrm{GeV} / \mathrm{cm}^{3}\right)$ & $r_{s}(\mathrm{kpc})$ & $\alpha$ \\
\hline 8 & 0.4 & 15.7 & 0.17
\end{tabular}

Table 1: Parameters used in the assumed dark matter density profiles (Eq. 3.1 and Eq. 3.2 ). $R_{\odot}$ and $\rho_{\odot}$ are respectively the distance from the sun to the Galactic center and the local dark matter density of the solar system and determine the scale density $\rho_{s}$. The scale radius $r_{s}$ is the radius such that $\rho\left(r_{s}\right)=\rho_{s}$ and $\alpha$ determines the logarithmic slope of the Einasto profile (the Burkert profile does not set the slope as a free parameter).

characterized by a sharp peak (cusp) towards the halo center [3] [4].

$$
\rho(r)=\rho_{s} e^{\frac{-2}{\alpha}\left[\left(r / r_{s}\right)^{\alpha}-1\right]}
$$

Here, $\rho_{s}$ is a normalization constant on the dark matter mass density determined by the total halo mass. $r_{s}$ is the characteristic scale radius of the halo and $\alpha$ determines the profile's curvature. The parameters chosen for our model are shown in Table 1.

Observations of dark matter halos may favor a flatter shape (core) towards the center. This is parameterized by the Burkert profile [5], shown in Eq. 3.2.

$$
\rho(r)=\frac{\rho_{s}}{\left(1+r / r_{s}\right)\left(1+\left(r / r_{s}\right)^{2}\right)}
$$

Here, $\rho_{s}$ and $r_{s}$ are again the density normalization and scale radius (see Table 1).

The difference in behavior of the profiles profiles is shown in Fig.2. At less than $10 \mathrm{kpc}$ from the Galactic Center, the predicted density differs by an order of magnitude or more between each profile, which introduces a substantial systematic uncertainty in the expected flux.

This uncertainty can be mitigated by considering a larger region of the Galactic halo further from the center, where the density profiles do not differ by as much. In the following sections, we present such an analysis performed with the HAWC detector.

\section{The HAWC Detector}

The High Altitude Water Cherenkov (HAWC) detector is a gamma-ray observatory located at Sierra Negra, Mexico. Consisting of an array of 300 water Cherenkov detectors and covering an area of $22,000 \mathrm{~m}^{2}$, it is able to detect gamma rays by the air showers they produce in the atmosphere. Air showers are created when charged particles or gamma rays interact with Earth's atmosphere, producing cascades of lower-energy particles. HAWC detects these secondary particles by the Cherenkov light they produce while passing through the water Cherenkov detectors, which is then detected with four photomultiplier tubes (PMTs) mounted at the bottom. Timing information is then used to reconstruct angle of arrival, and the distribution of charges is used to estimate energy [6].

The majority of air showers detected by HAWC come from charged cosmic rays. Therefore, cuts are applied to separate the hadronic events from the gamma rays before the data is analyzed. With cuts, 99\% of hadronic events are rejected at the highest energies [6]. However, the relative abundance of charged cosmic rays to gamma rays means the data is still dominated by background 


\section{Dark Matter Radial Profiles}

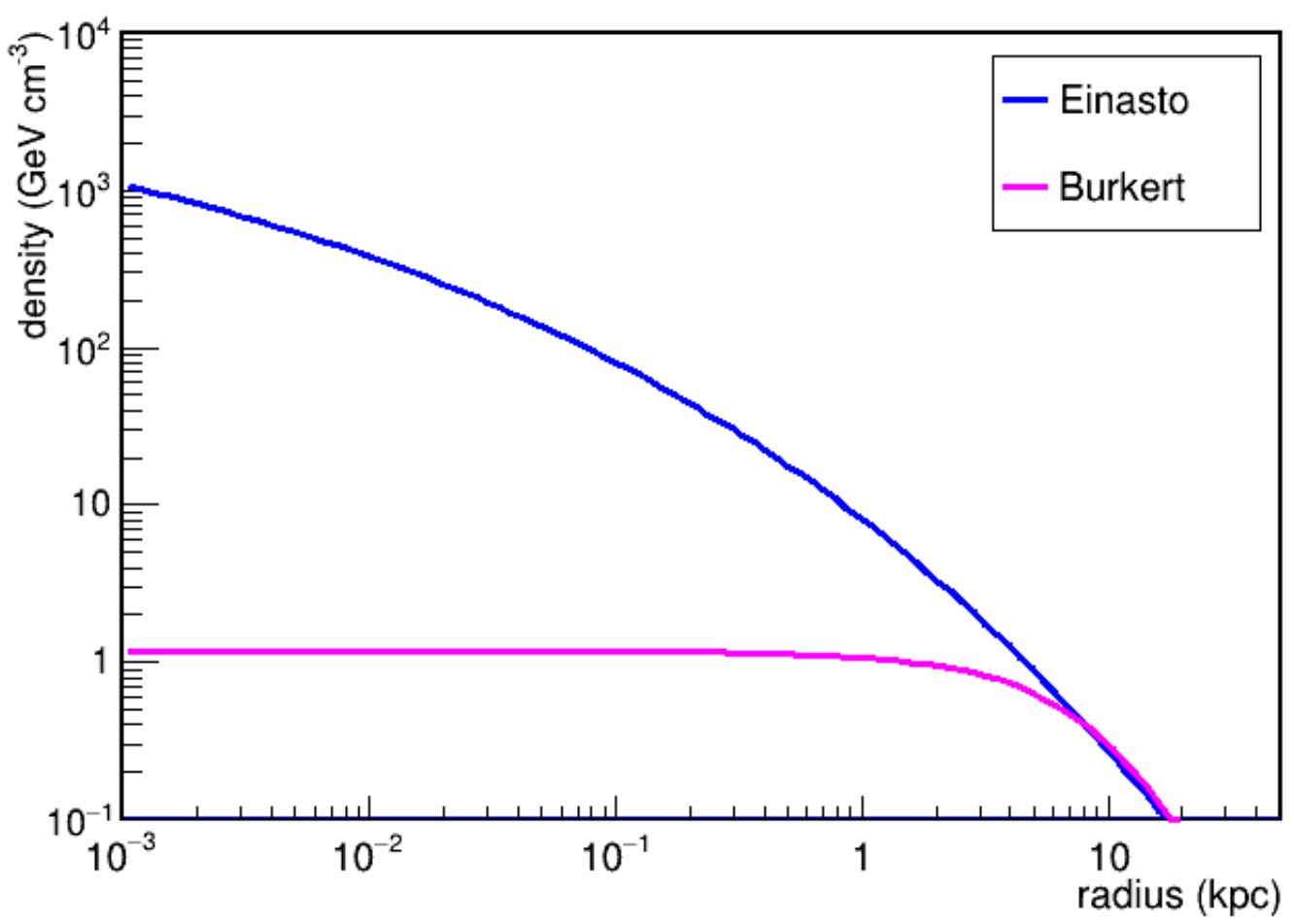

Figure 2: A comparison of the density profile behavior as a function of distance from the Galactic Halo center. The Einasto (cuspy) profile differs by nearly three orders of magnitude from the Burkert (cored) profile towards the center. The flux from regions further from the center is less dependent on the choice of density profile.

after cuts. To estimate the remaining background, we use a technique called direct integration (DI). Events are integrated within some time period in hour angle of a point to estimate the associated background [6]. The signal at each point in the sky is then the number of excess gamma rays above this estimated background level..

HAWC is sensitive to gamma rays above $500 \mathrm{GeV}$ and is well-suited for detecting signals from multi-TeV dark matter masses. In addition, HAWC operates on a near-continuous duty cycle with a wide field-of-view that makes it ideal for performing survey-style observations [6].

\section{Maps and Background Estimation}

This analysis uses 1038 days of HAWC data. The standard HAWC background estimation procedure would dampen any possible signal from the galactic halo. Direct integration assumes that events are background-dominated and localized to small regions of the sky, which is not true for flux from the galactic halo. To avoid overestimating the background, we extend the direct integration time to 24 hours (an entire strip of declination) and use a region of interest (ROI) mask. An ROI mask excludes any region of the sky expected to posses significant signal from the background estimate, in order to avoid upward biases. 
For our analysis, we chose as our ROI the region expected to yield the most sensitivity for HAWC. We estimate the HAWC sensitivity to dark matter as a function of declination by using the the procedure outlined in Ref [7]. Assuming a $b \bar{b}$ channel annihilation and a dark matter mass of $10 \mathrm{TeV}$, we find the characteristic (average) 95\% upper limit on $J\langle\sigma v\rangle$ at each declination in steps of 1 degree. Then, we associate a small point in the halo with a J-factor by integrating the density profile over a single pixel worth of solid angle ( 0.057 by 0.057 degrees). Using this, we obtain the expected upper limit on $\langle\sigma v\rangle$ from treating each pixel of the Galactic halo as a point source. Those points with the most constraining upper limits are then selected as part of ROI for the full extended source treatment. The resulting plots is shown for the Einasto and Burkert profiles are shown in Fig. 3.

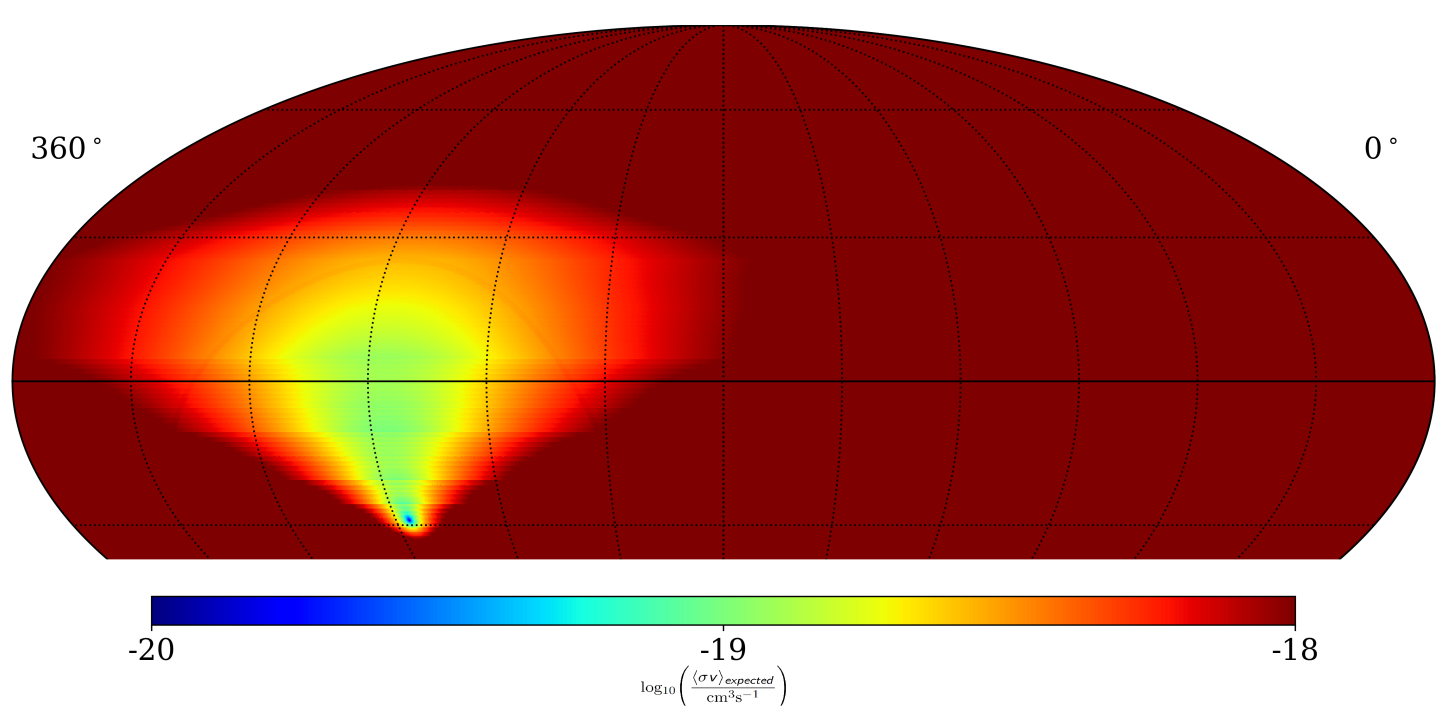

Figure 3: Expected upper limit at various locations in the Galactic halo obtained from treating each pixel as a point source and computing the corresponding J-factor. This figure assumes and Einasto profile with parameters given in Sec. 3, and demonstrates the HAWC sensitivity emission from the Galactic halo. Points with the most constraining upper limits will be included in the regions of interest for the full analysis and excluded from background estimation.

In estimating the background, we mask this ROI from the Galactic halo, as well as regions within 5 degrees of galactic latitude of the Galactic plane or any known luminous-matter sources of $\mathrm{TeV}$ gamma rays. The masked regions are plotted in Fig. 4

\section{Limits}

We compute a set of upper limits on dark matter from the Galactic halo using the standard HAWC maps. Due to the two hour direct integration and lack of an ROI mask, these maps are unable to resolve any excess originating from the extended Galactic halo. We instead use this data to estimate the upper limits that can be set in the case that the proper treatment of the background does not reveal any significant excess consistent with dark matter. 


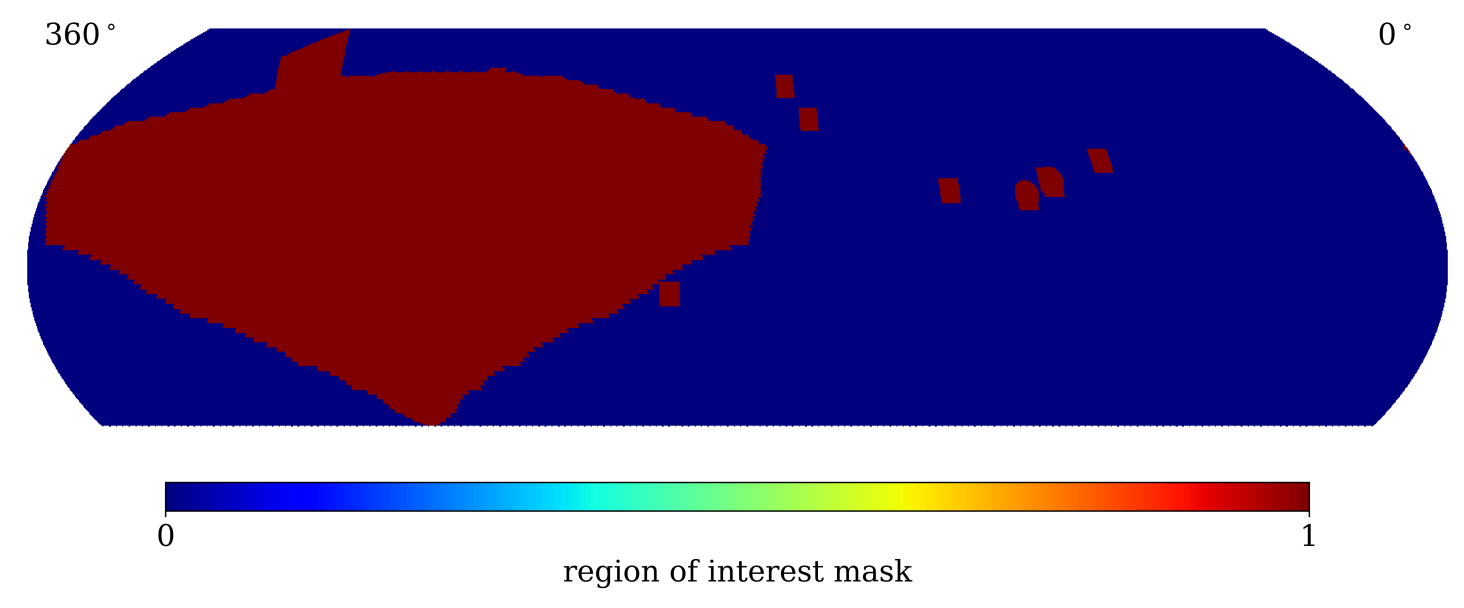

Figure 4: Regions masked (shown in red) when estimating the cosmic ray background. The region of the Galactic halo expected to yield the best sensitivity to dark matter for HAWC is masked, as well as any resolved sources known to originate from luminous matter. This prevents signal photons from biasing the background upward during direct integration.
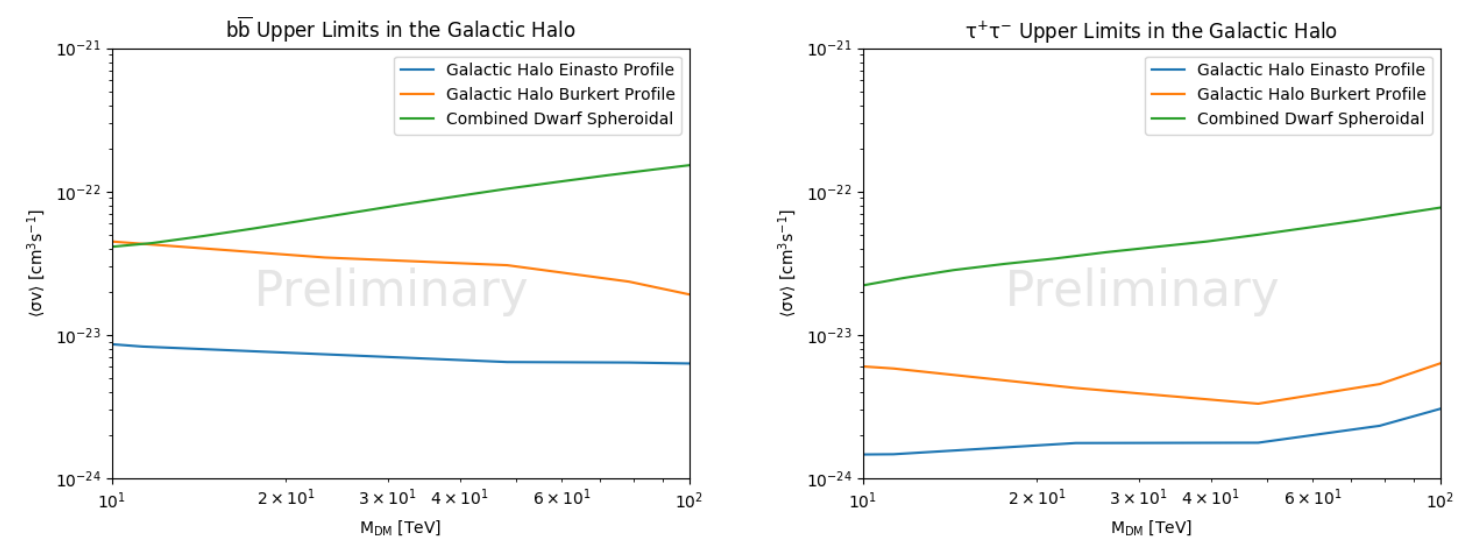

Figure 5: Estimated upper limits on dark matter annihilation in the Galactic halo using the standard HAWC dataset. The analysis used to make these plots is unable to resolve any possible excess originating from the dark matter halo, but is used to estimate the upper limits that will be set by the full analysis. 
The upper limits obtained are shown in Fig. 5. The limits are relatively insensitive to the choice of density profile, changing by only a factor of between two and three between the the cuspy (Einasto) and cored (Burkert) cases. We show results only for spectra with masses above $10 \mathrm{TeV}$. At lower energies, the small-scale cosmic ray anisotropy becomes a non-negligible effect and requires a more careful calculation of the background. A future analysis will take this effect into account as part of a larger effort to correctly calculate the background and search for possible excess originating from dark matter.

\section{Conclusion}

The HAWC observatory is ideal for probing highly extended sources. We build on previous work to optimize a dark matter search with HAWC in the Galactic halo. A region of interest is selected to optimize sensitivity to dark matter while being relatively insensitive to systematic uncertainties from the halo density profile. We then use this region of interest in combination with HAWC data to estimate the limits that can be set in this region. A new dataset is required to perform a proper search with the background recalculated to enable the possibility of detections.

\section{References}

[1] K. N. Abazajian and J. P. Harding, JCAP 1201 (2012) 041.

[2] T. SjÃústrand, S. Ask, J. R. Christiansen, R. Corke, N. Desai, P. Ilten, S. Mrenna, S. Prestel, C. O. Rasmussen, and P. Z. Skands, Comput. Phys. Commun. 191 (2015) 159-177.

[3] J. Stadel, D. Potter, B. Moore, J. Diemand, P. Madau, M. Zemp, M. Kuhlen, and V. Quilis, Mon. Not. Roy. Astron. Soc. 398 (2009) L21-L25.

[4] J. F. Navarro, A. Ludlow, V. Springel, J. Wang, M. Vogelsberger, S. D. M. White, A. Jenkins, C. S. Frenk, and A. Helmi, Mon. Not. Roy. Astron. Soc. 402 (2010) 21.

[5] A. Burkert, IAU Symp. 171 (1996) 175. [Astrophys. J.447,L25(1995)].

[6] A. U. Abeysekara et al., Astrophys. J. 843 (2017) 39.

[7] HAWC Collaboration, A. U. Abeysekara et al., arXiv:1811.11732. 